\title{
AVO waveform inversion for estimating the fluid contact with fluid transition zone
}

\author{
Yunosuke IWAKI $^{1}$, Hitoshi MIKADA ${ }^{1}$, Tada-nori GOTO ${ }^{1}$ and Junichi TAKEKAWA ${ }^{1}$ \\ ${ }^{1}$ Dept. of Civil and Earth Res. Eng., Kyoto University
}

\begin{abstract}
The fluid distribution in the hydrocarbon reservoir affects waveforms acquired in seismic reflection method. A reflected wave changes its waveform at the transition zone of the interface of two different fluids as a function of volume fraction of the two. AVO is in general used to estimate the difference in the $\mathrm{P}$ and $\mathrm{S}$ wave velocities for the interfacing two media at the interface without any assumptions on the existence of the transition zone. The consideration of the effect of the volume fraction of a fluid to the other in the waveform could be a key for evaluating the fluid mixture around the fluid contact in the reservoir. Therefore, we try to use the waveform directly to estimate fluid distribution in the transition zone that has not been done in the practice of AVO. In our research, we consider the effects of the transition zone at a gas-water contact (GWC) in a horizontally stratified medium on seismic waveforms. The numerical simulation reveals that the fluid distribution of transition zone distorts the seismic waveform both in amplitude and in phase. We developed a full-waveform AVO inversion method to apply to the fluid substitution problems to see if the method is applicable to estimate the fluid contact with the transition zone, while the conventional AVO only utilizes the amplitude derived from observed data. The simulation results imply the advantages in the estimation of the parameters including the thickness of the transition zone under that assumption of linear trend in the volume fraction in a contrast porosity condition. We suggest that the phase information should be used simultaneously for the inversion process to get the closer contact image.
\end{abstract}

\section{INTRODUCTION}

Time-lapse survey is one of the fluid monitoring methods using seismic reflection survey. The fluid distribution change in the hydrocarbon reservoir affects the observed waveform at the surface receivers. To estimate the fluid distribution, the attribute analysis is used with the observed data ${ }^{1)}$. This analysis applies a statistical process to the receiver data to evaluate the fluid property. We can use the relationship for estimating how the fluid moves with the oil and gas production. However, physical relationships between the fluid distribution and the observed waveform are not considered in this method.

The AVO inversion estimates the reflection contact in the subsurface reservoir. The conventional AVO inversion estimates the elastic parameters, P-wave velocity, S-wave velocity and density, with the maximum amplitude of the PP-reflected wave derived from the observed waveform data under the assumption of the horizontally stratified fluid contact. The fluid contact in the reservoir has a complicated fluid distribution and the fluid transition zone distorts the reflected waveform. Since this causes the misinterpretation for the evaluation of the hydrocarbon potential, the distortion of the reflected wave due to the fluid transition zone should be considered in the AVO inversion.

In this paper, we first confirm the effect of the fluid transition zone on the reflected waveform. Then we try to use the waveform difference for fluid contact estimation with a full-waveform AVO inversion based on the GLI method. We consider the effective magnitude of noise for reliable estimation using our AVO inversion method.

\section{THEORY}

\section{(1) Elastic and fluid parameter}

We simulate the wave propagation using an elastic finite-difference method. In order to derive the seismic waveform changed with the fluid alteration, we use the Gassmann's relation. The inclusion-based model can consider the fluid and solid phase as the elastic parameter. In this paper, we assume the fluid contact with a transition zone. The bulk modulus $K_{f}$ and density $\rho_{f}$ of fluid mixture is calculated by the Reuss and Voigt average of two fluid saturation ratios $f_{i}$. 


$$
\begin{aligned}
\frac{1}{K_{f}} & =\sum_{i=1}^{N} \frac{f_{i}}{K_{i}} \\
\rho_{\mathrm{f}} & =\sum_{\mathrm{i}=1}^{\mathrm{N}} f_{i} \mathrm{~d}_{\mathrm{i}}
\end{aligned}
$$

\section{(2) AVO inversion based on GLI method}

The GLI (Generalized Linear Inversion) method is an iterative inversion procedure which updates the calculation model parameter minimizing the misfit derived from the calculation and true model ${ }^{2)}$. We assume that the transition zone affects the seismic waveform. The AVO inversion uses the maximum amplitude, $A_{\theta}$ with the incident angle $\theta$, of PP-reflected wave. When the amplitude is changed by the elastic velocities and thickness of the transition zone, the misfit function $\mathrm{E}$ is calculated using the partial differential term,

$$
\mathrm{E}=\sum_{\theta}\left(\Delta \mathrm{A}_{\theta}-\frac{\partial \mathrm{A}_{\theta}}{\partial \mathrm{V}_{\mathrm{P} 2}} \Delta \mathrm{V}_{\mathrm{P} 2}-\frac{\partial \mathrm{A}_{\theta}}{\partial \mathrm{V}_{\mathrm{S} 2}} \Delta \mathrm{V}_{\mathrm{S} 2}-\frac{\partial \mathrm{A}_{\theta}}{\partial \mathrm{D}} \Delta \mathrm{D}\right)^{2}
$$

where $V_{P 2}$ and $V_{S 2}$ are the elastic velocities of the layer under the transition zone and $\mathrm{D}$ is the thickness of the transition zone. $\Delta \mathrm{A}_{\theta}$ means the subtraction of the amplitude of the true model and calculation model and $\Delta \mathrm{V}$ is the value of the model updating. The updating value is calculated for minimizing the misfit function by the least-square technique. In this paper, we assume that the transition zone condition is known such as fluid mixture law (determined by Reuss and Voigt average) and the linearity of fluid alteration.

\section{(3) Full-waveform AVO inversion}

The fluid transition zone mainly influences the change in the phase of the reflected wave. To use the time series data, we propose an advanced AVO inversion method. The misfit function using the amplitude of a certain time $t$ is written as below;

$$
\mathrm{E}=\sum_{\mathrm{t}} \sum_{\theta}\left(\Delta \mathrm{A}_{\theta, \mathrm{t}}-\frac{\partial \mathrm{A}_{\theta, \mathrm{t}}}{\partial \mathrm{V}_{\mathrm{P} 2}} \Delta \mathrm{V}_{\mathrm{P} 2}-\frac{\partial \mathrm{A}_{\theta, \mathrm{t}}}{\partial \mathrm{V}_{\mathrm{S} 2}} \Delta \mathrm{V}_{\mathrm{S} 2}-\frac{\partial \mathrm{A}_{\theta, \mathrm{t}}}{\partial \mathrm{D}} \Delta \mathrm{D}\right)^{2}(4)
$$

With the same procedure in the case of the maximum amplitude, we calculate the update parameter by minimizing the misfit function. In this method, we can use PP-reflected wave and PS-converted wave simultaneously.

\section{SIMULATION RESULT}

Figure 1 shows the calculation model used in this paper. The model is $4000 \mathrm{~m} \times 2000 \mathrm{~m}$ composed of shale in the upper layer and sandstone in the lower with a contact at the depth of $1500 \mathrm{~m}$. The shale is saturated with water. The sandstone is saturated with gas in $1500 \mathrm{~m}-1510 \mathrm{~m}$, and from the depth of $1510 \mathrm{~m}$, the transition zone begins at the gas-water contact (GWC). The gas is lineally alternated by water in this zone. The source and 60
Table 1 Rock property

\begin{tabular}{ccc}
\hline & Shale & Sandstone \\
\hline Bulk modulus $(\mathrm{GPa})$ & 10 & 35 \\
\hline Sheer modulus $(\mathrm{GPa})$ & 5.1 & 5.9 \\
\hline Density $\left(\mathrm{g} / \mathrm{cm}^{3}\right)$ & 2.6 & 2.6 \\
\hline Porosity & 0.1 & 0.2 \\
\hline
\end{tabular}

Table 2 Fluid property

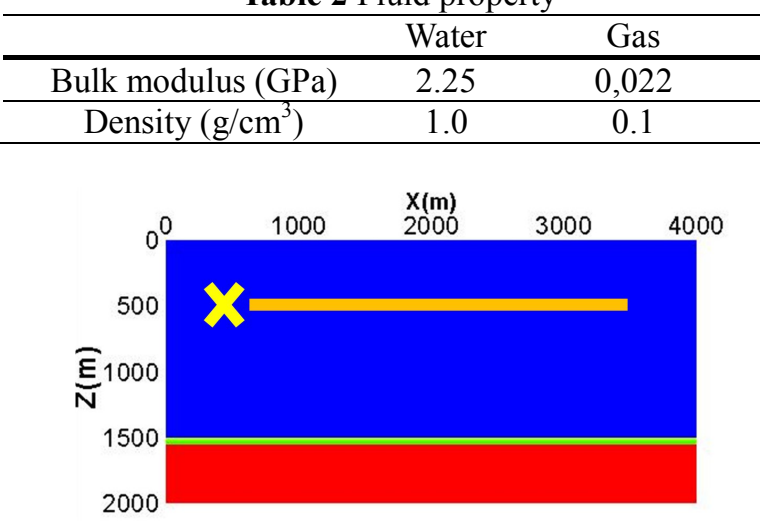

Figure 1 Calculation model: At the depth of $500 \mathrm{~m}, \mathrm{X}$ represents the source and a thick line is a receiver array. The contact of shale and sandstone is at the depth of $1500 \mathrm{~m}$.

receivers per $50 \mathrm{~m}$ are set at the depth of $500 \mathrm{~m}$. To ignore the effect of the reflected wave from the model boundaries, we assume the absorbing boundary at all the four edges. We used the rock and fluid properties shown in Table 1 and 2.

\section{(1) Wave form change with transition zone}

We obtained the waveform data from different transition zone model; model 1 has no transition zone, model 2 and 3 have the transition zone of $10 \mathrm{~m}$ and $40 \mathrm{~m}$ thickness, respectively. Figure 2 shows the maximum amplitude of PP-reflected wave observed at each receiver (the value is normalized by the amplitude of each incident wave). The amplitude changes at most $7 \%$ in model 2 and $35 \%$ in model 3 , compared with no transition zone model. Figure 3 shows the traveltime lag of the PP-reflected wave. We compared the lag of model 2 and 3 data subtracted from model 1 data. The traveltime delay is $3-5 \mathrm{~ms}$ in model 2 and $12-25 \mathrm{~ms}$ in model 3. Figure 4 shows the phase delay of waveforms observed by the model 1 and 3 in the frequency domain. The gradient of the figure represents the time delay.

The forward simulation shows that the transition at the GWC changes the reflected waveform. We can use this change for estimating the GWC property, elastic parameters and a thickness of transition zone by AVO inversion.

(2) Estimation of elastic parameters and thickness of the transition zone 


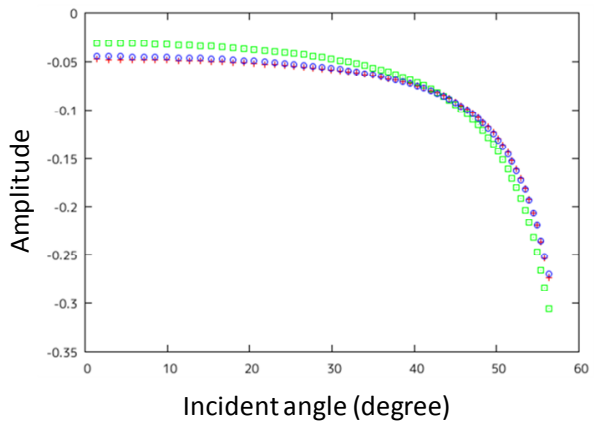

Figure 2 Amplitude changes by the offset (+: no transition zone, circle: $10 \mathrm{~m}$ thickness, square: $40 \mathrm{~m}$ thickness)

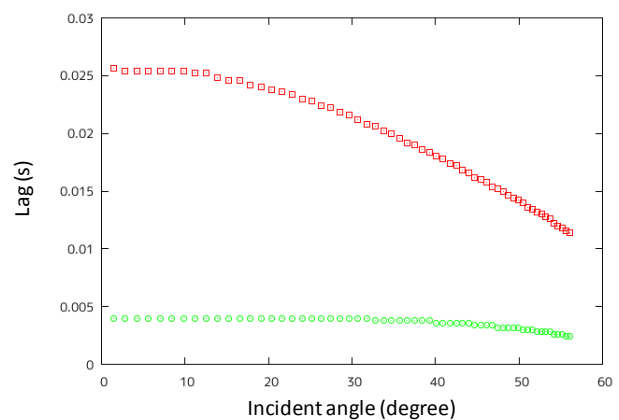

Figure 3 Time delay from the waveform of no transition zone model (circle: 10m thickness, square: $40 \mathrm{~m}$ thickness)

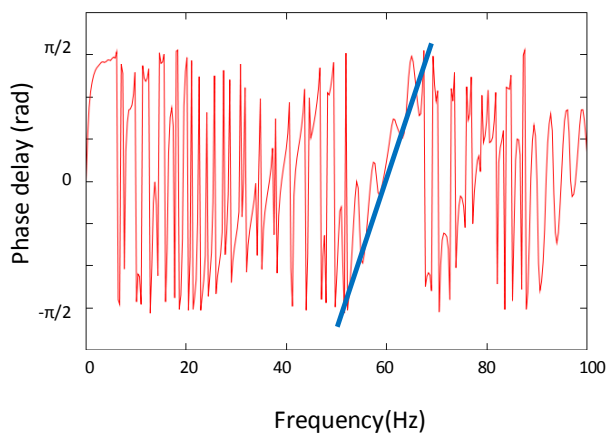

Figure 4 Phase delay between $0 \mathrm{~m}$ and $40 \mathrm{~m}$ thickness transition zone model in the frequency domain.

In chapter 2(3), we explained the AVO inversion with full-waveform. The reflected waveform has the effect from the GWC as the changes in the amplitude and phase, so we can apply the full-waveform AVO inversion to estimate the GWC. We applied our inversion method to the observed data derived from the $40 \mathrm{~m}$-thick transition zone model. The result (Table 3) shows the 3 estimated parameters are close to the true ones. Figure 5 shows the decrease of the misfit function. Figure 6 shows the waveform residuals between the data of the initial and true model (upper) and of the estimated and true model (lower). The residuals became almost zero by 8 iterations.

\section{(3) Estimation with the data including noise}

Table 3 AVO inversion result

\begin{tabular}{cccc}
\hline & $\begin{array}{c}\text { True } \\
\text { model }\end{array}$ & $\begin{array}{c}\text { Initial } \\
\text { model }\end{array}$ & $\begin{array}{c}\text { Inversion } \\
\text { result }\end{array}$ \\
\hline P-wave velocity $(\mathrm{m} / \mathrm{s})$ & 3126 & 3500 & 3127 \\
\hline S-wave velocity $(\mathrm{m} / \mathrm{s})$ & 1245 & 1267 & 1245 \\
\hline $\begin{array}{c}\text { transition zone } \\
\text { thickness }(\mathrm{m})\end{array}$ & 40 & 60 & 40 \\
\hline
\end{tabular}

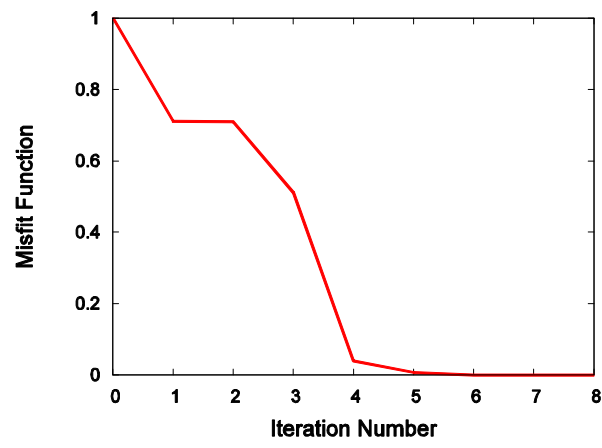

Figure 5 Misfit function
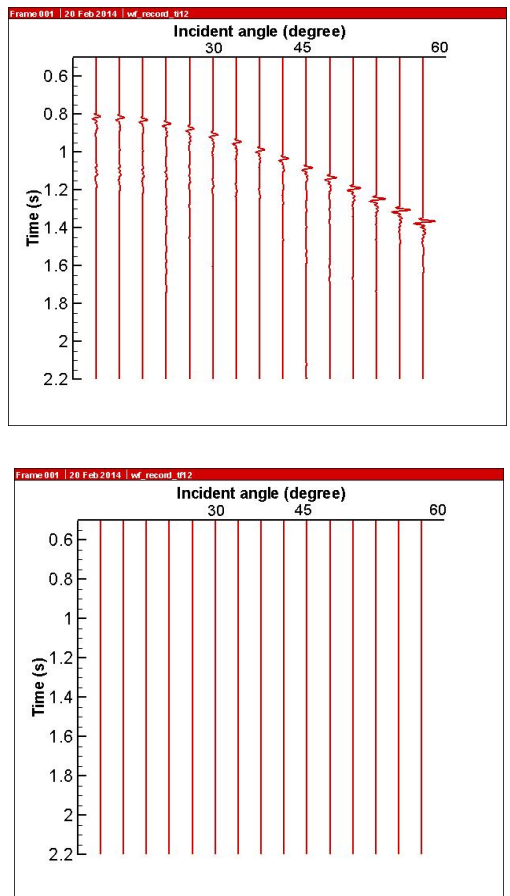

Figure 6 Waveform residuals subtracted by the observed (true) waveform: the initial (upper) and the inversion result (lower)

The AVO inversion using all waveform data has an advantage for applying it to the noise-included data. We apply the two AVO inversion methods, using the conventional PP-wave amplitude and all waveform to the data including $10 \%$ noise for the maximum amplitude at each receiver. Table 4 shows the inversion result. Although the conventional amplitude inversion estimated different value from the true parameters, the full-waveform AVO inversion estimated almost the same value. The increased amplitude with noise led to the wrong parameters shown in Figure 7. The full-waveform AVO inversion minimizes the misfit 
Table 4 AVO inversion result (data with $10 \%$ noise)

\begin{tabular}{cccc}
\hline & $\begin{array}{c}\text { True } \\
\text { model }\end{array}$ & $\begin{array}{c}\text { Amplitude } \\
\text { inversion }\end{array}$ & $\begin{array}{c}\text { full-wavefor } \\
\text { m inversion }\end{array}$ \\
\hline P-wave velocity $(\mathrm{m} / \mathrm{s})$ & 3126 & 3135 & 3126 \\
\hline S-wave velocity $(\mathrm{m} / \mathrm{s})$ & 1245 & 1240 & 1245 \\
\hline $\begin{array}{c}\text { transition zone } \\
\text { thickness }(\mathrm{m})\end{array}$ & 40 & 39 & 39 \\
\hline
\end{tabular}

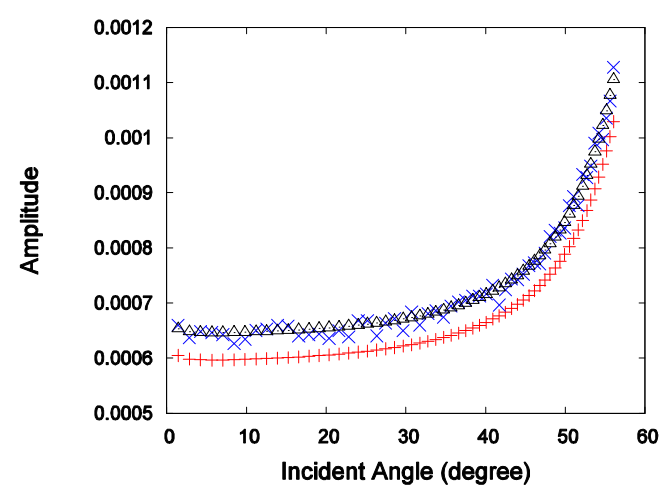

Figure 7 Amplitude per incident angle; +: observed data without noise, $\mathrm{X}$ : observed data with $10 \%$ noise, triangle: inversion result

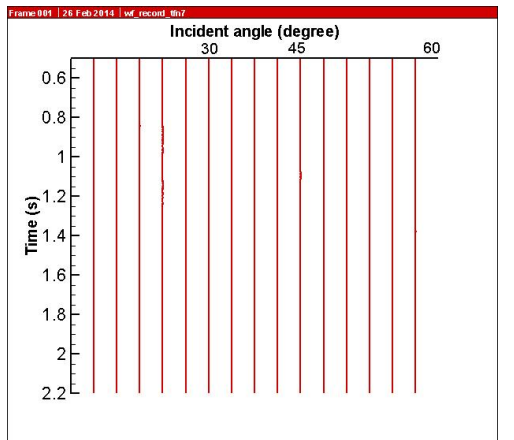

Figure 8 Waveform residuals of estimated subtracted by the observed waveform with $10 \%$ noise

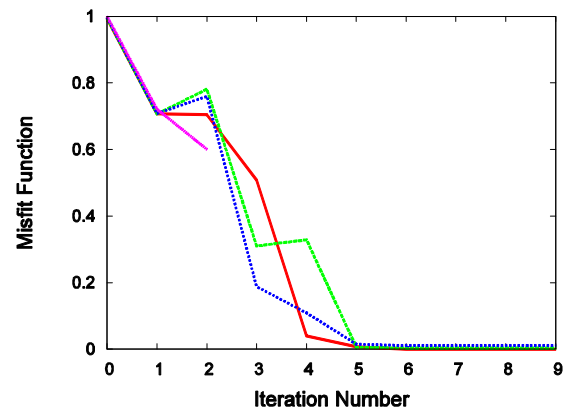

Figure 9 Misfit function in 3(3); solid line: noise free, long dashed line: $10 \%$ noise, dotted line: $20 \%$ noise, short dashed line: $24 \%$ noise (finished in 2 iterations)

function calculated by all receiver data, causing the better result (Figure 8). We also apply the full-waveform AVO inversion method to the data with different value of noise. We confirmed that the inversion is effective for $20 \%$ noise data, but in the case of $24 \%$ noise, the misfit function increased and the inversion didn't converge. We show the waveform residuals of the true with noise and
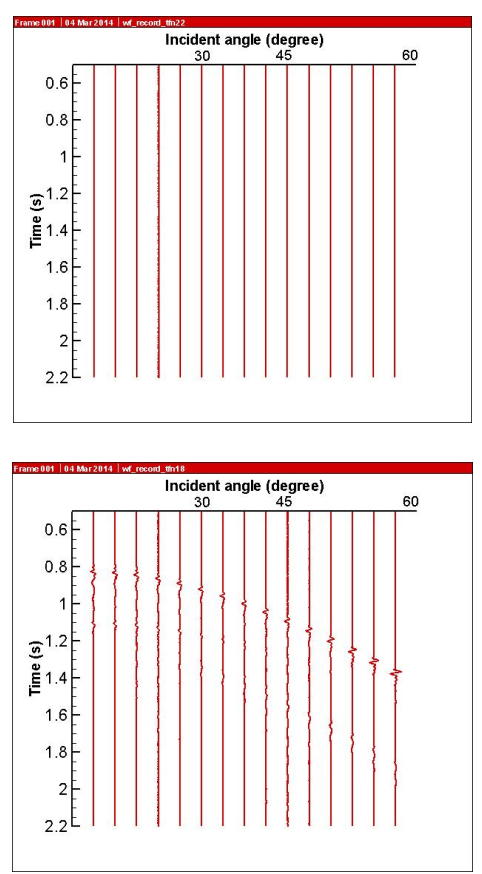

Figure 9 Waveform residuals of estimated subtracted by the true waveform with $20 \%$ (upper) and $24 \%$ (lower) noise.

estimated model in Figure 9.

\section{CONCLUSION}

We proposed an estimation method for the thickness of transition zone around a GWC and demonstrated the effectiveness of the method. The fluid distribution changing linearly with depth affects the amplitude and phase of the reflected waveform. Our AVO inversion for the full-waveform data can make best use of the whole change of the waveform. We applied the conventional amplitude method and full-waveform method to the synthetic data distorted by noise. The result showed that the full-waveform AVO inversion estimates closer value to the true model than the conventional one.

In this paper, we assumed an ideal model with stratified layers to focus on the confirmation of our AVO inversion method. It is necessary to apply this method to more complicated model such a case as the nonlinear fluid distribution in the transition zone or the fluid mixture law. The rock physics would assist this inversion method to estimate more complex reservoir.

\section{REFERENCES}

1) Taner, M. T., Koehler, F. and Sheriff, R. E., 1979, Complex seismic trace analysis, Geophysics, 44, 1041- 1063.

2) Cooke, D. A. and Schneider W. A., 1983, Generalized linear inversion of reflection seismic data, Geophysics, 48, 665-676. 